\title{
ESTRATEGIAS DE ENSEÑANZAS Y APRENDIZAJE EN LA EDUCACIÓN SUPERIOR FRENTE AL COVID-19
}

AUTORES: Delia Georgina Bravo Bonoso ${ }^{1}$

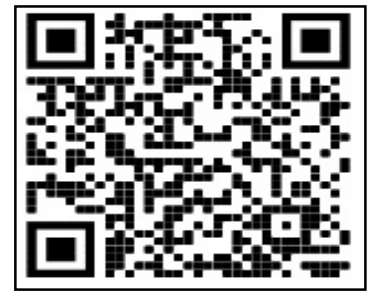

DIRECCIÓN PARA CORRESPONDENCIA: delia.bravo@unesum.edu.ec

Fecha de recepción: 01/08/2020

Fecha de aceptación: 15/09/2020

\section{RESUMEN}

Los efectos presentes de la crisis sobre la educación superior son fáciles de documentar con acciones que ha emprendido el gobierno, para garantizar el derecho a la educación superior durante la pandemia. La falta de referencias a crisis semejantes en el pasado hace difícil poder predecir qué pueda suceder en el futuro inmediato. En el caso de los estudiantes; el impacto inmediato ha sido, obviamente, el cese temporal de las actividades presenciales de las universidades, ha dejado a los que aspiran a ingresar a la educación superior, en una situación nueva y sin una idea clara de cuánto tiempo vaya a durar con impactos sobre su vida cotidiana, los costes soportados y sus cargas financieras y, por supuesto, la continuidad de sus aprendizajes. El profesorado sufre también importantes afectaciones en lo laboral y en lo profesional, hay que tomar en cuenta que no todas las IES tienen estrategias de continuidad de la actividad docente y, en su ausencia, los contratos temporales pueden quedar rescindidos. El personal no docente representa el sector más vulnerable en cuanto a la posible reducción de puestos de trabajo. Por lo tanto, el objetivo de este artículo es generar conciencia acerca de la importancia de los cambios educativos y pedagógicos que exige el mundo de hoy y el del futuro, teniendo en cuenta los avances tecnológicos, las diferencias en intereses, formas de aprender, habilidades de las nuevas generaciones y las consecuencias medioambientales que surgen de un mundo globalizado y que cada vez agotan las posibilidades de encuentros presenciales multitudinarios.

PALABRAS CLAVE: Pandemia, enseñanza, impacto, tecnologías.

\section{TEACHING AND LEARNING STRATEGIES IN HIGHER EDUCATION AGAINST COVID-19}

\section{ABSTRACT}

\footnotetext{
${ }^{1}$ Mg en Urgencias Médicas, Docente Investigador, Universidad Estatal del Sur de Manabí
} 
The current effects of the crisis on higher education are easy to document with the actions that the government has taken to guarantee the right to higher education during the pandemic. The lack of references to similar crises in the past makes it difficult to predict what might happen in the immediate future. In the case of students; The immediate impact has obviously been the temporary cessation of the universities' face-to-face activities, which has left those who aspire to enter higher education in a new situation and without a clear idea of how long it will last with impacts on their daily life, the costs incurred and their financial burdens and, of course, the continuity of their learning. The teaching staffs also suffer important effects in the workplace and professionally, it must be taken into account that not all HEIs have strategies for the continuity of teaching activity and, in their absence, temporary contracts may be terminated. Non-teaching personnel represent the most vulnerable sector in terms of possible reduction of jobs. Therefore, the objective of this article is to raise awareness about the importance of educational and pedagogical changes demanded by the world of today and that of the future, taking into account technological advances, differences in interests, ways of learning, skills of the new generations and the environmental consequences that arise from a globalized world and that each time exhaust the possibilities of massive face-to-face meetings.

KEYWORDS: Pandemic, teaching, impact, technologies.

\section{INTRODUCCIÓN}

A finales del mes de diciembre de 2019 se empiezan a registrar algunos casos de neumonía de origen desconocido en pacientes procedentes de Wuhan, provincia de Hubei (China). En enero de 2020 se pudo establecer la causa, una nueva cepa de un virus perteneciente a la familia Coronavirirusdae1 que tiene tropismo por las células del epitelio respiratorio2 y que al igual que las dos cepas anteriormente descritas, la cepa SARS (por sus siglas en inglés que traducen: Síndrome Respiratorio Agudo Grave), en 2003 y la cepa MERS (por sus siglas en inglés que traducen Síndrome Respiratorio de Oriente Medio), en 2012, se relaciona con una transmisión zoonótica y puede complicarse con neumonía en pacientes que presentan ciertas condiciones como edad avanzada o la presencia de comorbilidades (Ahmad et al., 2020).

Por lo anterior, el Gobierno de Ecuador, basado en experiencias de otros países como China, decidió implementar un plan de contención y mitigación de la infección por el COVID-19, pues el cierre de establecimientos públicos, restricción de movilidad en las fronteras y aislamiento temporal de la población en sus casas fue una estrategia que ayudó a disminuir considerablemente el número de casos en China a finales del mes de febrero del presente año. Estas medidas obviamente, involucraron al sector educativo, pues tanto Colegios como Universidades son lugares en los que pueden concentrarse un gran número de individuos lo que puede favorecer los procesos de transmisión, por lo tanto, tener control, registro y seguimiento diario del tránsito de cada uno de estos individuos además de sus contactos es casi imposible.

Considerando que pocos días después de confirmarse el primer caso en Ecuador, la cifra empezó a aumentar de forma significativa, el presidente de la República comunica que a partir del 16 de marzo de 2020 todas las entidades Públicas y educativas deben suspender sus clases para migrar a estrategias de educación virtual por lo menos hasta que se haya controlado la situación de salud y estado de emergencia, lo cual es incierto (Nacional, 2020). Lo anterior supone un desafío a la educación en Ecuador, sobre todo en áreas donde ni las instituciones ni los estudiantes cuentan 
con los recursos tecnológicos y/o cognitivos, así como la dificultad de adaptar la metodología de aprendizaje virtual para las personas en situación de discapacidad visual y/o auditiva, por lo que se hace cada vez más importante la formación de los docentes en estrategias de educación innovadoras, el uso pedagógico de las TICS (Tecnologías de la Información y de la Comunicación), el TPACK (Conocimiento Pedagógico y Tecnológico del Contenido) y la implementación de metodologías nuevas de enseñanza y aprendizaje.

Desde que la educación comenzó a centrarse en el alumno como el sujeto que aprende, se analiza a analizar no solo las tácticas que utiliza el profesor para desarrollar sus clases, sino también las estrategias de aprendizaje utilizado por los estudiantes. La enseñanza y el aprendizaje son procesos que se presentan juntos, es decir, las estrategias que se emplean para la instrucción inciden en los aprendizajes (Monereo \& Pozo, 2007), (Muñoz, 2003) que tienen los estudiantes tienen sus formas muy particulares de aprender.

El mes de marzo del 2020 se convirtió en un mes que América Latina no podrá olvidar debido a la suspensión de clases que ocurrió en casi todo el mundo como consecuencia directa de la cuarentena a resguardar por el COVID 19, donde el pánico colectivo, el estrés generado por el confinamiento y el rol de las instituciones educativas frente al uso de herramientas tecnológicas para crear ambientes de aprendizaje virtual improvisados, nos lleva a replantearnos el modo y la forma en que la escuela educa en tiempos de crisis.

Bajo la mirada crítica y reflexiva de la gestión administrativa de la educación, es necesario generar un abordaje académico que permita el acercamiento al modo y forma en que las instituciones educativas deberían prepararse para abordar las nuevas realidades que convergen alrededor de pandemias, guerras y el mismo calentamiento global. Es entonces cuando en medio de la crisis educativa provocada por el coronavirus, aparece con su aporte al mejor estilo de un discurso panegírico futurista, académicos de la talla de Edgar Morin quien en a finales de los años noventa publicaba su obra con el auspicio de la Organización de las Naciones Unidas para la Educación, la Ciencia y la Cultura (UNESCO) titulada "Los siete saberes necesarios para la educación del futuro"1 ya desde esos tiempos el brillante y celebre académico generaba con su excelsa obra bibliográfica una interesante reflexión sobre cómo formar para la consolidación de una educación que contribuya al futuro viable, un futuro demandante de crear aportes que den pauta a los cambios de pensamiento indispensables para preparar el porvenir de la educación ante tanta incertidumbre sobre el futuro educativo que les espera a las nuevas generaciones, sobre todo en estos tiempos tan difíciles para un mundo enfermo de COVID 19. MORIN, Edgar. Los siete saberes necesarios para la educación del futuro.

Morin sin saber lo que ocurriría en el 2020, ya vislumbraba tiempos caóticos que tendrían que demandar grandes cambios en los procesos de enseñanza y aprendizaje, por lo que al leer sus escritos es fácil encontrar sendos mensajes sobre como nuevos tiempos demandarían a los contextos educativos de una transformación fundamental que permita la mejora educativa en una sociedad global; y es que el COVID 19 dejo para el sector educativo una contundente evidencia que se necesitan cambios trascendentales en la forma como los docentes deben sobrellevar los nuevos escenarios que han surgido en consecuencia directa de una pandemia que provoca miseria

(C) Universidad Estatal del Sur de Manabí. Jipijapa, Ecuador. 
y dolor; todo ello condice a valorar el argumento de la Organización de Estados Iberoamericanos para la Educación quien calcula que en los 23 países de la comunidad iberoamericana miembros de la OEI, existen 14 millones de niños y niñas en el nivel infantil que han visto interrumpidas sus clases, 46 millones en educación primaria, 44 millones en secundaria y más de 18 millones de estudiantes universitarios, por lo que ante más de 122 millones de estudiantes afectados en Iberoamérica.

\section{DESARROLLO}

La gran pregunta es ¿están las instituciones educativas preparadas para afrontar las crisis generadas ante situaciones como la ocasionada por el COVID 19?

Edgar Morin sentenciaba que, frente a nuevas realidades sociales, el adecuar cambios a la educación ayudara en buena medida a fortalecer el proceso de enseñanza y aprendizaje como un reto desafiante que permita dar cara a la complejidad de los hechos sociales llenos de cambios repentinos y desconocidos. Lo anterior significa que, en un contexto postmodernista y propio del siglo XXI, se deben modificar las maneras de enseñar y aprovechar los recursos informáticos para organizar el conocimiento, lo cual implica reformular políticas y programas en materia educativa con el fin de que sea el docente quien facilite una motivación en el estudiante para que este adquiera conductas conscientes, que impliquen el reconocimiento de acciones como la autocrítica, la automotivación y una férrea voluntad de resolver problemas, de tal forma que cuando fenómenos como el COVID 19 vuelvan a perjudicar al mundo, las nuevas generaciones sepan que las implicaciones de un cambio en tiempos de crisis, involucran una contextualización social de las situaciones críticas para sacar el mejor provecho de ellas.

El primer saber (afrontar las incertidumbres) estipula que en innumerables momentos y ante infinidad de situaciones, hemos sentido incertidumbre frente a lo inesperado, y es que precisamente lo incierto suele darle al hecho educativo una perspectiva de inseguridad y vulnerabilidad que desboca el desequilibrio e inestabilidad. Ante esto, Morin cuestiona ¿por qué no enseñar principios de estrategias que permitan afrontar los riesgos?, y es que de alguna manera, el académico antes mencionado, asume que dando respuesta a la pregunta vertida, se puede buscar que los docentes puedan enfrentar lo inesperado y modificar el desarrollo de su actuar formativo con base en las informaciones previamente adquiridas y planeadas; potenciando que el buen docente deba siempre tener en mente varias alternativas de solución a los problemas, procurando cada vez más el empoderarse de la certeza que frente a hechos como el coronavirus se debe actuar buscando agotar la incertidumbre.

La suspensión de clases demanda en forma urgente de una experiencia en la docencia virtual, ya que en muchas latitudes geográficas, este forma de abordar una clase haciendo uso del internet nos acerca al punto de lo importante que es saber afrontar las incertidumbres, sobre todo cuando el cierre de las instituciones educativas muestra en forma acentuada la abismal diferencia entre los estudiantes urbanos más acomodados, que cuentan con móviles y ordenadores con una buena conexión a Internet, y aquellos con menor capacidad y que no que no cuentan con teléfonos celulares ni computadoras, peor aún, una conexión a internet que les permita continuar las clases virtuales en plataformas como Zoom, Moddle o Hangouts de Google,Meet, las cuales destacan entre las favoritas de los docentes y muchas instituciones. 
Las universidades centroamericanas por su parte, también ofrecen su aporte frente a las crisis pandémica actual, y es que en la mirada analítica del académico Oscar Picardo de nacionalidad uruguaya y en su calidad de director del Instituto de Ciencia, Tecnología e Innovación de la Universidad Francisco Gavidia, argumenta que algunas universidades, poseen unidades de modelaje matemático que podrían colaborar en tiempos de coronavirus, recomendando a los diversos gobiernos de los países más golpeados por la pandemia a recurrir al uso de un modelaje matemático que determine una métrica centrada en datos y cifras que orienten a epidemiólogos, biólogos, médicos y científicos matemáticos al servicio de la salud pública, a enfrentar con eficiencia y eficacia la mejora de una acción educativa en tiempos de crisis. Y es que, en base al argumento del doctor Picardo, se podría llegar al plano de que, con cálculos incorrectos y sin ciencia, el mundo corre el riesgo de terminar con buena salud, pero con la economía agonizando, por lo que no se debe obviar que la primera vacuna que la sociedad necesita ante el caos provocado por el COVID 19 es la vacuna educativa y científica (Pericado, 2020).

A parte del modelaje matemático, muchas instituciones educativas de todos los niveles de enseñanza, se han lanzado a canalizar el hecho formativo mediante el uso de plataformas digitales que contribuyen a enfrentar los riesgos y la incertidumbre de perder el año escolar y no darle el normal abordaje al contenido curricular de sus asignaturas, por lo que ya son muchos los profesores en el mundo que recurriendo a breves capacitaciones teóricas y algunas prácticas, han podido impartir su clase desde cualquier rincón del planeta. El agregar mejores recursos tecnológicos al acto educativo debe favorecer en todas formas nuevos medios de aprendizaje que apegados al hecho colaborativo y cooperativo permitan sobrellevar las distintas situaciones en un momento donde el internet ayuda a que los estudiantes afectados por el coronavirus a no detener su potencial por aprender.

El segundo saber (enseñar la comprensión) Le da la oportunidad a Edgar Morin de retomar el uso de la reflexión a fin de considerar por qué razón en pleno siglo XXI, se tienen muestras de desprecio, racismo y xenofobia en el contexto educativo, llevando a la pregunta de ¿Dónde queda la comprensión en nuestros tiempos de crisis? Siendo esta (la comprensión) un medio y un fin de la comunicación humana, y siendo el principio de las relaciones humanas, sigue percibiéndose la marcada ausencia de una acción educativa que empodere la comprensión como centro del debate.

Las diversas acciones llevadas a cabo por los docentes en sus diversas prácticas desde la virtualidad ante el coronavirus, les ayudará a mejorar sus capacidades y habilidades, desarrollando sus competencias con sacrificio, pero con mucha creatividad.

La UNESUM decide suspender las actividades educativas, administrativas y sugerir la migración hacia estrategias de aprendizaje virtual. Esto supone un desafío para la institución, además de un reto para los profesores, pues se hace necesario un cambio en las metodologías de enseñanza y aprendizaje; se vuelve urgente el tema de innovación pedagógica, pues se requiere, en poco tiempo, adaptar las metodologías utilizadas hasta el momento, para migrar al ambiente virtual. Los tiempos de sosobra que vive una sociedad durante una Pandemia, pueden ser positivos para la educación pues deben llevar a los maestros a la reflexión, a la necesidad de formación en

(C) Universidad Estatal del Sur de Manabí. Jipijapa, Ecuador. 
competencias pedagógicas y en el dominio del conocimiento pedagógico y tecnológico del contenido.

La innovación pedagógica que se tiene que llevar a cabo en los tiempos del Coronavirus podrá derribar muros, tumbar mitos, creencias y lograr generar en los profesores la motivación para realizar el cambio educativo que necesita la generación de jóvenes que estamos formando. Por tanto, el propósito de éste artículo es generar conciencia acerca de la importancia de los cambios educativos y pedagógicos que exige el mundo de hoy y el del futuro, teniendo en cuenta los avances tecnológicos, las diferencias en intereses, formas de aprender, habilidades de las nuevas generaciones y las consecuencias medioambientales que surgen de un mundo globalizado en el que cada vez serán más complejas las posibilidades de encuentros presenciales multitudinarios.

Tabla 1. Actividades diseñadas para cada uno de los pasos del PPI (Plan Pedagógico Institucional) en el ambiente de enseñanza y aprendizaje presencial y su adaptación al ambiente virtual.

\begin{tabular}{|c|c|c|}
\hline Pasos del PPI & $\begin{array}{c}\text { Actividades diseñadas en metodología } \\
\text { de Aula Invertida }\end{array}$ & $\begin{array}{l}\text { Adaptación de Actividades para Modalidad 100\% } \\
\text { virtual }\end{array}$ \\
\hline Contexto & $\begin{array}{l}\text { 1. Guía de Lectura: ruta y orientación de la } \\
\text { preparación del tema, apoyado con las } \\
\text { referencias recomendadas por los profesores } \\
\text { 2. Vídeos explicativos: el profesor explica } \\
\text { con ayuda de gráficas, tablas y figuras } \\
\text { aquellos conceptos más complejos. }\end{array}$ & $\begin{array}{l}\text { 1. Guía de Lectura: ruta y orientación de la preparación del } \\
\text { tema, apoyado con las referencias recomendadas por los } \\
\text { profesores. 2. Vídeos explicativos: el profesor explica con } \\
\text { ayuda de gráficas, tablas y figuras aquellos conceptos más } \\
\text { complejos. }\end{array}$ \\
\hline Experiencia & $\begin{array}{l}\text { Actividades que involucran dimensiones } \\
\text { cognitivas y emocionales como, entrevistas a } \\
\text { pacientes que han presentado una infección } \\
\text { que hace parte del tema de clase, búsqueda } \\
\text { del mapa de la infección, Práctica en el } \\
\text { Hospital Simulado con casos de pacientes } \\
\text { simulando infecciones virales respiratorias, } \\
\text { análisis de casos clínicos, etc. }\end{array}$ & $\begin{array}{l}\text { Actividades que involucran dimensiones cognitivas y } \\
\text { emocionales adaptadas a los ambientes virtuales como: Análisis } \\
\text { de casos clínicos que pueden ser compartidos por el aula virtual, } \\
\text { tour virtual por el Hospital simulado, laboratorios virtuales de } \\
\text { observación de estructuras bacterianas, micóticas o parasitarias, } \\
\text { vídeos interactivos acerca de la patogénesis de los diferentes } \\
\text { microorganismos, fragmentos de películas que muestran las } \\
\text { consecuencias clínicas y epidemiológicas de las infecciones, así } \\
\text { como encuentros sincrónicos por los cuales el profesor } \\
\text { comparte presentaciones y material relacionado. }\end{array}$ \\
\hline Reflexión & $\begin{array}{l}\text { Actividades diseñadas para generar procesos } \\
\text { de reflexión e introspección de los saberes: } \\
\text { foros de discusión, exposiciones de casos } \\
\text { clínicos, clubes de revistas con artículos que } \\
\text { abordan el tema, actividades lúdicas como } \\
\text { juegos de preguntas o dramatización de casos } \\
\text { clínicos. }\end{array}$ & $\begin{array}{l}\text { Actividades diseñadas para generar procesos de reflexión e } \\
\text { introspección de los saberes adaptadas a los ambientes virtuales } \\
\text { como: foros virtuales de discusión, exposiciones de casos } \\
\text { clínicos, clubes de revistas con artículos que abordan el tema, } \\
\text { actividades lúdicas como juegos de preguntas y conexión a } \\
\text { través de encuentros sincrónicos utilizando herramientas como } \\
\text { Classroom u otras que favorecen la interacción entre alumnos y } \\
\text { profesores por chats o video conferencias }\end{array}$ \\
\hline Acción & $\begin{array}{l}\text { Fase en la que el estudiante debe dar cuenta } \\
\text { que ha adquirido el conocimiento del tema y } \\
\text { que ha alcanzado los objetivos de } \\
\text { aprendizaje: Se realiza por medio de } \\
\text { actividades que pueden ser presenciales como } \\
\text { pruebas escritas con casos o problemas del } \\
\text { tema o actividades que pueden ser virtuales a } \\
\text { través de la incorporación de herramientas } \\
\text { electrónicas virtuales como zoom, meet. }\end{array}$ & $\begin{array}{l}\text { Fase en la que el estudiante debe dar cuenta que ha adquirido el } \\
\text { conocimiento del tema y que ha alcanzado los objetivos de } \\
\text { aprendizaje, en el caso de la adaptación hacia actividades } \\
\text { virtuales se han diseñado pruebas en Classroom que permiten } \\
\text { evaluar el alcance de los objetivos, análisis de casos y se } \\
\text { continúa utilizando el apoyo de herramientas interactivas. }\end{array}$ \\
\hline Evaluación & $\begin{array}{l}\text { Este es un proceso trans Classroom } \\
\text { versal durante todos los pasos del } \\
\text { proceso de aprendizaje, pues cada uno } \\
\text { de los momentos tiene una calificación } \\
\text { que permite que el estudiante tenga un } \\
\text { indicador de su proceso de aprendizaje } \\
\text { y para el profesor es muy valioso } \\
\text { porque permite llevar un seguimiento } \\
\text { continuo e individualizado del } \\
\text { estudiante. La evaluación se realiza con } \\
\text { Rúbricas que los estudiantes conocen } \\
\text { incluso desde antes de iniciar las } \\
\text { actividades, lo que hace que el proceso } \\
\text { sea transparente. }\end{array}$ & $\begin{array}{l}\text { Al igual que en el modelo presencial, en el modelo virtual se } \\
\text { mantiene la importancia de la evaluación transversal durante } \\
\text { todos los pasos del proceso de aprendizaje, para que el } \\
\text { estudiante tenga un indicador de su rendimiento y para que el } \\
\text { profesor pueda tener el seguimiento continuo e individualizado } \\
\text { del estudiante. La evaluación se realiza igualmente con } \\
\text { Rúbricas que los estudiantes conocen incluso desde antes de } \\
\text { iniciar las actividades, lo que hace que el proceso sea } \\
\text { transparente. }\end{array}$ \\
\hline
\end{tabular}

126 UNESUM-Ciencias. Publicación cuatrimestral. Vol. 4, No. 3 (Número Especial), Año 2020. 
Los cambios tecnológicos tienen un impacto inevitable en la educación. Sin embargo, con una u otra tecnología es imprescindible preguntarse sobre nuevas formas de enseñar y aprender, nuevas concepciones pedagógicas y metodológicas. El contexto digital puede facilitar el camino a la innovación docente, siempre y cuando haya el diseño y la mediación del profesorado.

La educación no escapa del punto crítico que debe llevar a la reflexión, sobre todo cuando un virus obligó a profesores y maestros a salir de su zona de comodidad para empezar a diseñar estrategias de enseñanza y aprendizaje diferentes a la clase magistral, lo cual es interesante porque puede darle sentido a la necesidad de formación en pedagogía y en el conocimiento pedagógico y tecnológico del contenido, que llevará necesariamente a derribar muros, mitos, creencias y actitudes cimentadas en la costumbre arraigada de transmitir conceptos a una generación que ya no requiere estas formas de aprender y que necesita que se le dé respuesta a sus propias necesidades.

La organización y estructura del planeamiento o syllabus de una asignatura facilita la adaptación hacia ambientes de enseñanza y aprendizaje virtual, en ese sentido el Plan Pedagógico es el método que ayuda y orienta al docente en el "como" lograr la migración hacia la educación virtual, considerando que el profesor debe constituirse en un tutor que acompañe, guíe y oriente al estudiante y que pueda mantener una disponibilidad permanente para evitar la deserción y mantener la motivación en el proceso de enseñanza y aprendizaje. La innovación pedagógica en los tiempos del Coronavirus favorece la continuidad del proceso educativo y del cronograma académico de instituciones, maestros y estudiantes y puede ser el punto de partida para el cambio que el mundo exige desde hace muchos años.

Posiblemente estemos ante la generación de un nuevo paradigma educativo. Lo anterior podría llevarnos a pensar que la tragedia de una pandemia puede dejarnos aprendizajes y situaciones que nos llevan a ver realmente la causa de los problemas que aquejan a nuestra sociedad y a nuestros discentes, tal como lo expresó Gabriel García Márquez en "El amor en los tiempos del Cólera" (Márquez, 2003).

A pesar de que se cuenta con diagnósticos de los impactos del cierre de las Instituciones de Educación Superior, las experiencias emprendidas en otros países a raíz del confinamiento por la contingencia derivada de la aparición del COVID-19 y a nivel nacional se establecieron, a mediados de abril, recomendaciones o lineamientos para transitar a la docencia en línea en el nivel superior, es evidente que lo súbito de la situación y el cambio de modalidad, hicieron insuficiente la capacidad de comunicación, coordinación y respuesta de las instituciones, lo que no ha hecho posible que se ponga a disposición de las comunidades educativas las estrategias, herramientas y recursos necesarios para hacer frente a la transición de modalidad de manera oportuna y en mejores condiciones, decir que lo prioritario es concluir el Periodo Academico es negar la afectación en la consecución de los objetivos de aprendizaje, por lo que se requerirá no dejar de implantar medidas de nivelación.

\section{Materiales y Métodos}


Esta investigación es de tipo cualitativa, con un diseño de tipo descriptivo y exploratorio, desde una perspectiva teórico-metodológica de fenomenología, ya que se basa en la descripción de vivencias o experiencia subjetiva frente a un fenómeno. Además, se indagó sobre los recursos utilizados y las estrategias pedagógicas implementadas por los docentes en la modalidad de educación virtual impuesta por la pandemia.

Se elaboró un cuestionario semiestructurado que contempla algunos test, preguntas cerradas y abiertas. Se recolectaron datos sobre variables de base (edad, sexo, cargo que ocupa, etc.). Por otra parte, se indagó sobre los nuevos escenarios docentes, uso de tecnologías, los recursos y estrategias utilizados en los espaciosvirtuales.

\section{Resultados y Discusión}

La situación de confinio y la demanda de educación en línea, se les solicitó a los participantes que indicarán con qué frecuencia utilizaban alguna de las tecnologías propuestas para realizar su tarea pedagógica con sus estudiantes. El instrumento permitió puntuar a cada tecnología mediante una escala Likert de 5 puntos (nunca $=0$ a siempre $=4$ ). Para una mejor interpretación de los datos, se convirtieron los valores a escala 10 obteniendo los siguientes resultados.

Tabla 2.Tecnología utilizada

\begin{tabular}{|l|c|}
\hline \multicolumn{1}{|c|}{ Tecnologías } & Puntaje \\
\hline a) Plataformas virtuales (Moodle, Classroom,etc.) & 4,17 \\
\hline b) Aula virtual & 2,66 \\
\hline c) Videoconferencias (Zoom, Meet,etc.) & 4,16 \\
\hline d) Grupos WhatsApp & 8,83 \\
\hline e) Foros de debate online & 2,11 \\
\hline f) Redes sociales ( Instagram, Facebook,etc.) & 3,54 \\
\hline g) Elaboración de Blog o páginas web con contenido de estudio & 2,24 \\
\hline h) Distribución de material impreso & 2,47 \\
\hline
\end{tabular}

Fuente: elaboración propia

Como puede observarse en la tabla precedente, la mayor puntuación de frecuencia de uso es para la tecnología WhatsApp con 8,83 pts., despegándose significativamente del resto de las tecnologías. Respecto del material impreso (2,47 pts.), los docentesllevan a las casas de los estudiantes la documentación en formato papel para que éstos lo trabajen, esta situación es más propia de las zonas rurales donde no hay conectividad para los alumnos.

Tabla 3. Lista de recursos pedagógicos digitales

\begin{tabular}{|l|l|}
\hline Tecnologías & Puntaje \\
\hline a) Dictado de clases online & 5,23 \\
\hline b) Grabación digital de clases & 5,61 \\
\hline c) Digitalización de recursos didácticos preexistentes & 7,40 \\
\hline d) Elaboración de guías de estudio digitales & 7,27 \\
\hline e) Evaluación a distancia de los contenidos curriculares & 5,92 \\
\hline f) Presentaciones estilo PowerPoint & 5,33 \\
\hline
\end{tabular}

128 UNESUM-Ciencias. Publicación cuatrimestral. Vol. 4, No. 3 (Número Especial), Año 2020. 
Fuente: elaboración propia

Las estrategias pedagógicas más destacadas son la digitalización de documentos (7,4 pts.) y la elaboración de guías de estudio (7,27 pts.). En último lugar se encuentra el dictado de clases online (5,23 pts.) que se caracteriza por ser uno de los recursos más demandantes de conectividad $\mathrm{y}$ ancho de banda.

\section{Rendimiento académico y apoyo familiar}

Se les solicitó también a los participantes que emitieran su opinión respecto del rendimiento académico de sus estudiantes, teniendo en cuenta esta situación atípica parala educación. Además, se les invitó a que estimaran el grado de apoyo y contención por parte de los padres o familiares con los que conviven. Los resultados obtenidos son los siguientes:

Tabla 4. Rendimiento académico de los estudiantes y apoyo familiar

\begin{tabular}{|l|l|l|l|}
\hline & & Porcentaje & Total \\
\hline & Nulo & $0,90 \%$ & \\
\hline Rendimiento & Por debajo de lo normal & $48,91 \%$ & \multirow{2}{*}{$100 \%$} \\
\hline Académico & Normal & $47,36 \%$ & \\
\hline & Por encima de lo normal & $2,83 \%$ & \\
\hline & Sin apoyo & $7,85 \%$ & \\
\hline Apoyo familiar & Por debajo de lo esperado & $31,40 \%$ & \multirow{2}{*}{$100 \%$} \\
\hline & Normal & $42,73 \%$ & \\
\hline & Por encima de lo esperado & $18,02 \%$ & \\
\hline
\end{tabular}

Fuente: elaboración propia

Figura 4. Rendimiento académico de los estudiantes

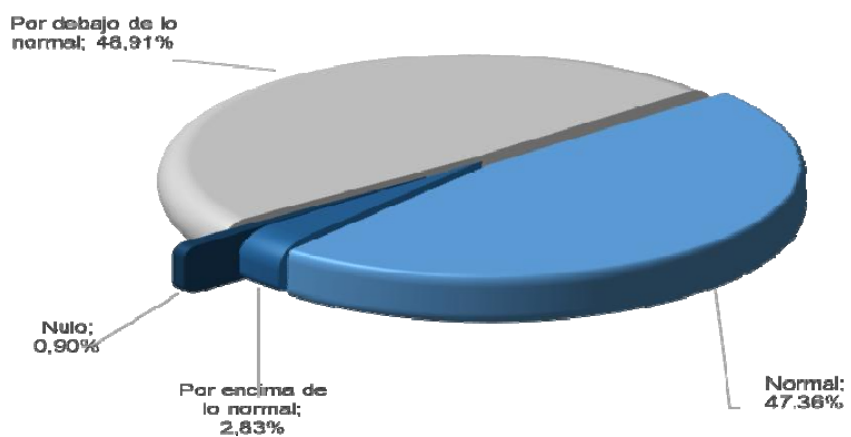

Fuente: elaboración propia 
En términos generales se puede decir que la muestra se dividió en dos mitades, una parte $(48,91 \%)$ sostiene que el rendimiento de sus alumnos está por debajo de lo normal y la otra parte $(47,36 \%)$ plantea que el rendimiento es normal. Los que consideran que el rendimiento se encuentra por encima de lo normal, solo cubren el 2,83\% de la muestra y los que consideran que es nulo apenas llegan al 0,9\%.

Respecto del nivel de apoyo familiar, el 42,73\% de los docentes estiman que la normalidad se ha mantenido. Casi el tercio (31,4\%) plantea que en nivel de apoyo es inferior al normal y solo el $7,85 \%$ dice que no existe apoyo familiar. Un 18,02\% de pedagogos resalta que el apoyo superó sus expectativas.

Para un análisis más detallado se relacionaron estas dos variables con el nivel educativo donde el docente lleva a cabo su tarea pedagógica. Los resultados son los siguientes.

Figura 5. Apoyo familiar de los estudiantes

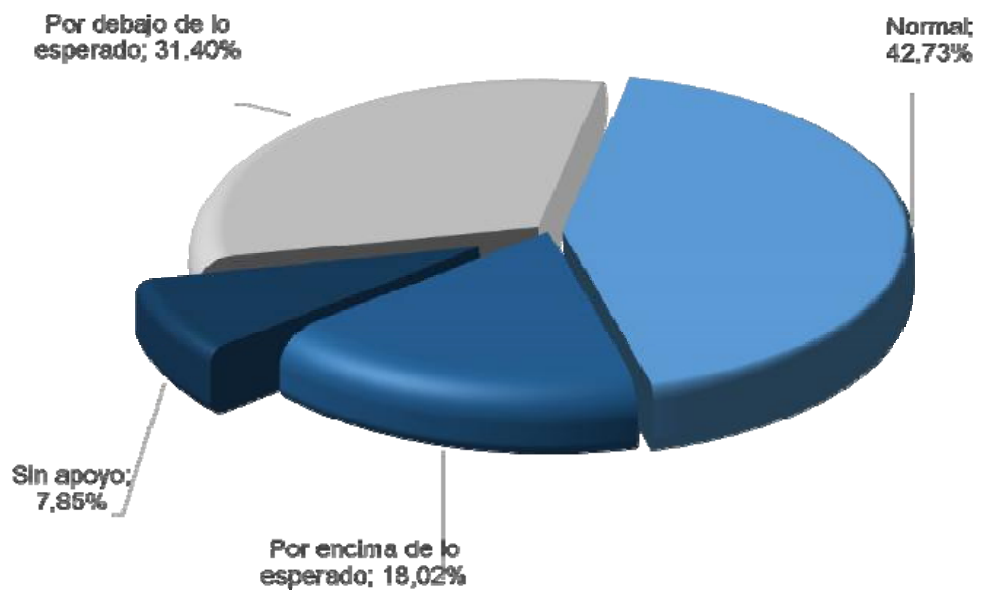

Fuente: elaboración propia

Tabla 5. Rendimiento académico de los estudiantes y apoyo familiar

\begin{tabular}{lcc}
\hline & & Univ. \\
\cline { 2 - 3 } Rendimiento Académico & Nulo & $0,0 \%$ \\
& Bajo & $38,1 \%$ \\
& Normal & $57,1 \%$ \\
Apoyo familiar & Alto & $4,8 \%$ \\
& Sin apoyo & 16,70 \\
& Bajo & $\%$ \\
& Normal & $9,50 \%$ \\
& Alto & 64,30 \\
& & $9,50 \%$ \\
\hline
\end{tabular}

Fuente: elaboración propia

130 UNESUM-Ciencias. Publicación cuatrimestral. Vol. 4, No. 3 (Número Especial), Año 2020. 


\section{CONCLUSIONES}

Los profesores universitarios sostienen que el acompañamiento es normal y también inexistente. Por último, es necesario resaltar que los docentes de educación especial expresan que este soporte familiar está por debajo de sus expectativas.

Con relación a la implementación de recursos pedagógicos digitales se pudo observar que, al ser tan precipitada esta situación de confinamiento social, los docentes se volcaron a la digitalización de material didáctico preexistente y la elaboración de guías de estudio. A su vez se identificaron dos tipos de recursos (Documentos y Clases) a los que se redujeron los indicadores utilizados. Se pudo constatar que el uso de las clases online como recurso pedagógico está mayormente empleado por los docentes, en alumnos de niveles socioeconómicos medios y altos.

En síntesis, los resultados obtenidos confirman las primeras aproximaciones efectuadas en otros contextos, que dan cuenta de que la situación de pandemia puso en evidencia la desigualdad de oportunidades educativas, las diferencias entre quienes tuvieron mejor acceso a los recursos tecnológicos y a internet; las diferencias en el capital cultural de las familias.

\section{REFERENCIAS BIBLIOGRÁFICAS}

Ahmad, T., Khan, M., Haroon, T. H. M., Nasir, S., Hui, J., Bonilla-Aldana, D. K., \& Rodriguez-Morales, A. J. (2020). COVID-19: Zoonotic aspects. Travel Medicine and Infectious Disease. https://www.ncbi.nlm.nih.gov/pmc/articles/PMC7128549/

Márquez, G. G. (2003). El amor en los tiempos del cólera. Vintage Español. http://www.geocities.ws/libros/LIBROS\%201/NOVELAS/ANOR\%20EN\%20TIENPOS\%20DE\%20COLER A/colera.pdf

Monereo, C., \& Pozo, J. I. (2007). Competencias para (con) vivir con el siglo XXI. Cuadernos de pedagogía, 370(12), 12-18. http://maaz.ihmc.us/rid=1GLSWYC7N-Y2LLHHH7H/Competencias\%20para\%20convivircon\%20el\%20siglo\%20XXI.pdf

Muñoz, R. F. (2003). Competencias profesionales del docente en la sociedad del siglo XXI. Organización y gestión educativa: Revista del Fórum Europeo de Administradores de la Educación,

Nacional C. Presidencia de la Republica del Ecuador. [Online]. Quito; 2020 [cited 202005 21. Available from: https://www.defensa.gob.ec/wp-content/uploads/downloads/2020/03/Decreto_presidencial_No_1017_17Marzo-2020.pdf

Pericardo OC. El Diario de hoy. [Online]; 2020 [cited 202003 25. Available from: https://www.elsalvador.com/eldiariodehoy/oscarpicardo-coronavirus-opinion/698890/2020/

(C) Universidad Estatal del Sur de Manabí. Jipijapa, Ecuador. 